\title{
The impact of trans-catheter aortic valve replacement induced left-bundle branch block on cardiac reverse remodeling
}

Laura E. Dobson', Tarique A. Musa', Akhlaque Uddin', Timothy A. Fairbairn', Owen J. Bebb ${ }^{1}$, Peter P. Swoboda', Philip Haaf', James Foley ${ }^{1}$, Pankaj Garg' ${ }^{1}$, Graham J. Fent ${ }^{1}$, Christopher J. Malkin², Daniel J. Blackman², Sven Plein ${ }^{1,2}$ and John P. Greenwood ${ }^{1,2^{*}}$

\begin{abstract}
Background: Left bundle branch block (LBBB) is common following trans-catheter aortic valve replacement (TAVR) and has been linked to increased mortality, although whether this is related to less favourable cardiac reverse remodeling is unclear. The aim of the study was to investigate the impact of TAVR induced LBBB on cardiac reverse remodeling.
\end{abstract}

Methods: 48 patients undergoing TAVR for severe aortic stenosis were evaluated. 24 patients with new LBBB (LBBB-T) following TAVR were matched with 24 patients with a narrow post-procedure QRS (nQRS). Patients underwent cardiovascular magnetic resonance (CMR) prior to and $6 \mathrm{~m}$ post-TAVR. Measured cardiac reverse remodeling parameters included left ventricular (LV) size, ejection fraction (LVEF) and global longitudinal strain (GLS). Inter- and intra-ventricular dyssynchrony were determined using time to peak radial strain derived from CMR Feature Tracking.

Results: In the LBBB-T group there was an increase in QRS duration from $96 \pm 14$ to $151 \pm 12 \mathrm{~ms}(P<0.001)$ leading to inter- and intra-ventricular dyssynchrony (inter: LBBB-T $130 \pm 73$ vs nQRS $23 \pm 86$ ms, $p<0.001$; intra: LBBB-T $118 \pm 103$ vs. nQRS $13 \pm 106 \mathrm{~ms}, p=0.001$ ). Change in indexed LV end-systolic volume (LVESVi), LVEF and GLS was significantly different between the two groups (LVESVi: nQRS $-7.9 \pm 14.0 \mathrm{vs}$. LBBB-T $-0.6 \pm 10.2 \mathrm{ml} / \mathrm{m}^{2}$, $p=0.02$, LVEF: nQRS $+4.6 \pm 7.8$ vs LBBB-T $-2.1 \pm 6.9 \%, p=0.002 ; \mathrm{GLS}: \mathrm{nQRS}-2.1 \pm 3.6$ vs. LBBB-T $+0.2 \pm 3.2 \%, p=0.024)$. There was a significant correlation between change in QRS and change in $\operatorname{LVEF}(r=-0.434, p=0.002)$ and between change in QRS and change in GLS $(r=0.462, p=0.001)$. Post-procedure QRS duration was an independent predictor of change in LVEF and GLS at 6 months.

Conclusion: TAVR-induced LBBB is associated with less favourable cardiac reverse remodeling at medium term follow up. In view of this, every effort should be made to prevent TAVR-induced LBBB, especially as TAVR is now being extended to a younger, lower risk population.

Keywords: Aortic valve stenosis, Trans-catheter aortic valve implantation, Left bundle branch block, Ventricular ejection fraction, Ventricular remodeling, Cardiovascular magnetic resonance

\footnotetext{
* Correspondence: j.greenwood@leeds.ac.uk

${ }^{1}$ Multidisciplinary Cardiovascular Research Centre (MCRC) \& Leeds Institute of

Cardiovascular and Metabolic Medicine (LICAMM), University of Leeds,

Clarendon Way, Leeds LS2 9JT, UK

${ }^{2}$ Department of Cardiology, Leeds Teaching Hospitals NHS Trust, Leeds LS1

3EX, UK
} 


\section{Background}

The aortic valve lies close to the electrical conduction system of the heart and is prone to damage at the time of aortic valve intervention, often manifesting as new left-bundle branch block (LBBB). New LBBB is infrequent following surgical aortic valve replacement [1], but much more common following trans-catheter aortic valve replacement (TAVR) with reported rates of up to $65 \%$, depending on valve design [2]. TAVR-induced left-bundle branch block (LBBB-T) has been linked to reduced survival [3-5] and increased hospitalisation [6], in keeping with population based studies suggesting reduced overall survival in healthy individuals with LBBB [7] and in patients with heart failure and LBBB [8]. The mechanism for this increased mortality is debated; one hypothesis is that LBBB-T is a precursor to further more lethal conduction abnormalities [9], another is that LBBB-T leads to abnormal left ventricular (LV) remodeling and ultimately heart failure death via a LBBB-induced cardiomyopathy [10]. Current evidence on the impact of LBBB-T on cardiac reverse remodeling is limited to echocardiographic studies, with a heterogeneous patient mix including those with post-procedural permanent pacemaker implantation, trans-apical access route and unmatched patient groups [10-12], all of which are potential confounders in the reverse remodeling process. The impact of LBBB-T on cardiac reverse remodeling has never been investigated using cardiovascular magnetic resonance (CMR), which is the reference standard technique for LV mass and volume quantification, allowing important differences to be determined with a small sample size [13]. Furthermore, the novel technique of feature tracking allows accurate estimation of global longitudinal strain (GLS) and inter- and intraventricular dyssynchrony which are of interest in this population and may be able to assess the impact of LBBB on cardiac function beyond simple mechanical dyssynchrony [14].

We hypothesised that LBBB-T 1) negatively impacts on cardiac reverse remodeling at $6 \mathrm{~m}$ follow up and 2) is associated with inter- and intra-ventricular dyssynchrony compared with a matched 'control' population with a narrow QRS (nQRS) post-TAVR.

\section{Methods}

\section{Patient selection}

We evaluated 88 patients undergoing either Boston Lotus (Boston Scientific Corporation, Natick, MA) or Medtronic CoreValve (Medtronic Inc., Minneapolis, Minnesota) TAVR for severe symptomatic aortic stenosis at a single tertiary centre from April 2009 to April 2015. Exclusion criterion included pre-existing QRS prolongation ( $>120 \mathrm{~ms}$ ) or contra-indication to CMR scanning. Decision for TAVR was taken by a multi-disciplinary heart team in accordance with international guidance [15]. Trans-femoral was the default approach with other techniques (subclavian and carotid) employed if femoral access was unsuitable.

\section{Matching}

24 patients with LBBB-T were identified. These were matched with 24 patients with a nQRS post-procedure for sex, valve type, and baseline CMR variables known to impact on reverse remodeling following TAVR including LV ejection fraction (LVEF), indexed LV mass and indexed LV end diastolic volume (LVEDVi) [16] (Fig. 1).

\section{Electrocardiographic data}

12-lead electrocardiogram recordings acquired immediately prior to TAVR and at the time of post-procedure hospital discharge were reviewed by a single author blinded to clinical and procedural data. Heart rhythm, PR interval and QRS duration were recorded. LBBB-T was defined as post-procedural v1-negative QRS complex with a duration of $>120 \mathrm{~ms}$ and a notched or slurred $\mathrm{R}$ wave in at least one of the lateral leads (I, aVL, $\mathrm{V}_{5}, \mathrm{~V}_{6}$ ) [17].

\section{CMR protocol}

Details of the CMR pulse sequence acquisition protocol have been published previously [16]. Briefly, identical CMR scans were obtained at baseline and $6 \mathrm{~m}$ following TAVR using a $1.5 \mathrm{~T}$ scanner (Intera, Philips Healthcare, Best, Netherlands or Avanto, Siemens Medical Systems, Erlangen, Germany). Multi-slice, multi-phase cine imaging was performed using a standard steady-state free precession pulse sequence in the short axis $(10 \mathrm{~mm}$ thickness, $0 \mathrm{~mm}$ gap, 30 phases, 192 by 192 matrix, typical field of view $340 \mathrm{~mm}$ ) to cover both ventricles. Standard 2, 3 and 4 chamber cine images were also acquired. Through-plane velocity encoded phase contrast imaging was performed perpendicular to the aortic valve jet at the aortic sinotubular junction (VENC 250$500 \mathrm{~cm} / \mathrm{s}$, retrospective gating, slice thickness $6 \mathrm{~mm}, 40$ phases). Late gadolinium enhancement (LGE) imaging (10-12 short axis slices, $10 \mathrm{~mm}$ thickness, matrix $240 \times 240,320-460 \mathrm{~mm}$ field of view) was performed with inversion time individually adjusted according to TI scout, $10-15 \mathrm{~min}$ after $0.2 \mathrm{mmol} / \mathrm{kg}$ of gadoteric acid (Dotarem, Guerbet, Villepinte).

\section{CMR analysis}

CMR analysis was performed by a single experienced operator blinded to clinical data using $\mathrm{cmr}^{42}$ (Circle Cardiovascular Imaging, Alberta, Canada). Endocardial and epicardial contours were manually contoured at end-diastole and end-systole with papillary muscles and trabeculations excluded to allow the calculation of 


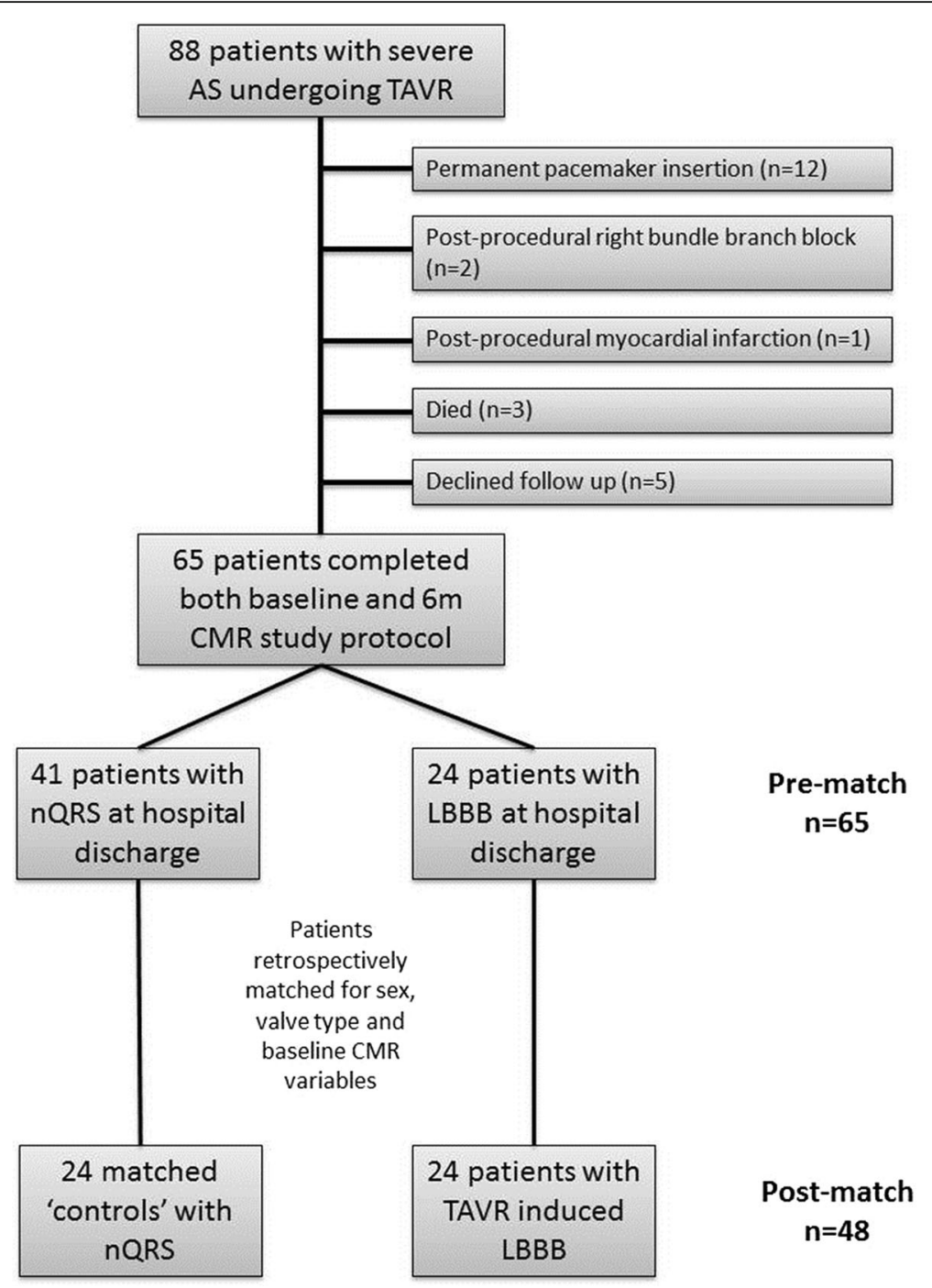

Fig. 1 Patient recruitment and retrospective matching methodology. AS: Aortic stenosis. TAVR: Trans-catheter aortic valve replacement. CMR: Cardiovascular magnetic resonance. nQRS: Narrow QRS. LBBB: Left bundle branch block

ventricular volumes (summation of discs methodology) and mass (epicardial volume - endocardial volume multiplied by myocardial density $\left.\left(1.05 \mathrm{~g} / \mathrm{cm}^{3}\right)\right)$. Values were indexed to body surface area. Post-procedural myocardial infarct was determined by direct comparison of pre- and $6 \mathrm{~m}$ CMR LGE acquisitions. Fibrosis mass was quantified using a threshold of 5 standard deviations technique [18]. Left atrial volume was calculated using the biplane area-length method [19]. Aortic flow was quantified using cross-sectional phase contrast images with the slice positioned at least $10 \mathrm{~mm}$ above the aortic prosthesis and contouring of the aortic lumen to provide a regurgitant fraction (\%). Longitudinal right ventricular function was measured at the lateral tricuspid annulus in the 4 chamber cine view as the distance between end systole and end diastole. Feature tracking analysis was performed on cine imaging of the mid ventricular short axis slice at the papillary muscle level to determine time to peak LV and right ventricular radial strain and the 4 chamber cine to measure global longitudinal strain. Interventricular dyssynchrony was the difference between time to peak radial strain of the right ventricular free wall and the lateral LV wall (an average of segments 11 and 12 of the American Heart Association 17 segment model). Intraventricular dyssynchrony was the difference between time to peak radial strain of the LV septal (an average of segments 8 and 9) and lateral LV segments (an average of segments 11 and 12). Segments with LGE indicating previous myocardial infarction were excluded from radial strain analysis. For the assessment of inter-observer variability, two independent investigators analysed LV volume, mass and function, GLS and time to peak radial strain on a random selection of 10 
patients. For intra-observer variability a similar dataset from 10 patients was analysed twice by one investigator one month apart. The coefficient of variation was calculated by dividing the standard deviation of the differences between measurements by their mean and expressed as a percentage. Inter-observer variability was $1.4,4.5,3.7,9.2$ and $12.6 \%$ and intra-observer variability was $6.8,2.6,5.0,2.6 \%, 6.8$ and $9.1 \%$ for LVEDV, LV mass, LVEF, GLS and time to peak radial strain respectively.

\section{Statistical analysis}

Statistical analysis was performed using SPSS version 22 (IBM, Armonk, New York). Categorical data were presented as numbers (percentages) and compared using the Pearson Chi squared test. Continuous variables were expressed as mean $\pm \mathrm{SD}$ and were tested for normality using the Shapiro-Wilks test. Data were compared using Students $t$ Test (for normally distributed data) and the Mann-Whitney or Wilcoxen signed rank test (for nonnormally distributed data). Linear regression analysis (Enter model) was performed to establish univariate and multivariate predictors of change in LVEF and GLS postprocedure. Univariate predictors with $P<0.05$ were included in the multivariate analysis. $P<0.05$ was considered statistically significant. Using CMR, in order to detect a $3 \%$ difference in LVEF with a $90 \%$ power and an $\alpha$ error of 0.05 a sample size of at least 12 patients in each arm was required [13].

\section{Results}

Eighty-eight patients were recruited. Patients undergoing post-procedure permanent pacemaker implantation $(n=12)$, those with post-procedure right bundle branch block $(n=2)$ and those with CMR LGE evidence of post-procedural myocardial infarction $(n=1)$ were excluded from analysis. In addition, 3 patients died within the $6 \mathrm{~m}$ follow up period and 5 patients declined follow up (Fig. 1). 24 patients with LBBB-T and 41 patients with nQRS on discharge electrocardiogram completed both baseline (median 1 day pre-procedure, IQR 1 day) and $6 \mathrm{~m}$ scans (median 181 days, IQR 20 days) and were available for retrospective matching in a 1:1 fashion (Fig. 1). 48 patients were included in the final analysis, 24 with LBBB-T and 24 with nQRS. Demographic, clinical, procedural and baseline CMR details for each group are shown in Table 1. 14 (29\%) patients underwent Lotus valve and 34 (71\%) patients underwent Medtronic CoreValve implantation. Balloon valvuloplasty was performed in $43(90 \%)$ patients. Mean valve size was $28 \pm 2 \mathrm{~mm}$, procedure time $164 \pm 52 \mathrm{mins}$ and contrast dose $153 \pm 61 \mathrm{ml}$. Access approach was femoral in 43 (90\%) patients, subclavian in $4 .(8 \%)$ patients and carotid in one patient.
Table 1 Demographic, clinical and baseline CMR details of the nQRS and LBBB-T groups

\begin{tabular}{|c|c|c|c|}
\hline & $\begin{array}{l}\mathrm{nQRS} \\
(n=24)\end{array}$ & $\begin{array}{l}\text { LBBB-T } \\
(n=24)\end{array}$ & $P$ value \\
\hline \multicolumn{4}{|l|}{ Demographic details } \\
\hline Age, years & $80.5 \pm 6.2$ & $79.6 \pm 9.6$ & 0.670 \\
\hline Gender, male & $13(54)$ & $13(54)$ & 1 \\
\hline Body surface area, $\mathrm{m}^{2}$ & $1.82 \pm 0.29$ & $1.86 \pm 0.19$ & 0.332 \\
\hline \multicolumn{4}{|l|}{ Clinical details } \\
\hline STS PROM, \% & $4.5 \pm 2.4$ & $5.1 \pm 2.8$ & 0.397 \\
\hline STS Morbidity/mortality, \% & $21.7 \pm 7.5$ & $24.5 \pm 8.8$ & 0.452 \\
\hline Systolic blood pressure, $\mathrm{mmHg}$ & $134 \pm 25.9$ & $138 \pm 18$ & 0.558 \\
\hline Hypertension & $12(57.1)$ & $9(37.5)$ & 0.383 \\
\hline Cerebrovascular disease & $4(16.7)$ & $4(16.7)$ & 1 \\
\hline Previous myocardial infarction & $5(20.8)$ & $2(8.3)$ & 0.220 \\
\hline $\begin{array}{l}\text { Chronic obstructive pulmonary } \\
\text { disease }\end{array}$ & $6(25)$ & $5(20.8)$ & 0.731 \\
\hline Peripheral vascular disease & $6(25)$ & $7(29.2)$ & 0.745 \\
\hline Diabetes mellitus & $4(16.7)$ & $8(33.3)$ & 0.182 \\
\hline $\begin{array}{l}\text { Any epicardial coronary stenosis } \\
>50 \%\end{array}$ & $9(37.5)$ & $13(54.2)$ & 0.247 \\
\hline \multicolumn{4}{|l|}{ Procedural details } \\
\hline Medtronic CoreValve & $17(71)$ & $17(71)$ & 1 \\
\hline Pre-implant valvuloplasty & $22(92)$ & $21(88)$ & 0.637 \\
\hline Post-implant valvuloplasty & $6(25)$ & $6(25)$ & 1 \\
\hline Femoral access site & $20(83)$ & $23(96)$ & 0.331 \\
\hline \multicolumn{4}{|l|}{ CMR data } \\
\hline Fibrosis mass, $g$ & $3.3 \pm 5.7$ & $1.6 \pm 3.8$ & 0.081 \\
\hline
\end{tabular}

Data are expressed as mean \pm SD or number (\%). STS PROM: Society of

thoracic surgeons predicted risk of mortality

\section{Electrocardiographic characteristics}

Mean heart rate at baseline was $67 \pm 11$ and at $6 \mathrm{~m}$ was $68 \pm 13 \mathrm{bpm} .7$ patients (15\%) (nQRS $n=2$, LBBB-T $n=5$ ) had atrial fibrillation at baseline. There were no new cases of post-procedural atrial fibrillation. For those in sinus rhythm, mean PR interval remained similar pre and post procedure in both the nQRS group $(179 \pm 33$ to $191 \pm$ $39 \mathrm{~ms}, p=0.053)$ and the LBBB-T group (181 \pm 30 to 192 $\pm 37 \mathrm{~ms}, p=0.171$ ). In the nQRS group there was no change in QRS duration ( $93 \pm 17$ to $96 \pm 11 \mathrm{~ms}, p=0.098$ ). In the LBBB-T group, QRS duration increased from $96 \pm$ 14 to $151 \pm 12 \mathrm{~ms}(p<0.001)$.

\section{Reverse remodeling according to post-procedure QRS duration}

Change in LVEF and GLS was significantly different between the two groups (LVEF: nQRS $+4.6 \pm 7.8$ vs LBBB$\mathrm{T}-2.1 \pm 6.9 \%, p=0.002$ and GLS: nQRS $-2.1 \pm 3.6$ vs. LBBB-T $+0.2 \pm 3.2 \%, p=0.024$ ) (Fig. 2). The change in 




LVEF was driven by a reduction in indexed LV end systolic volume (LVESVi) in the nQRS group not seen in the LBBB-T group (nQRS $-7.9 \pm 14.0$ vs. LBBB-T $-0.6 \pm$ $10.2 \mathrm{ml} / \mathrm{m}^{2}, p=0.02$ ). Pre and post-procedure values for all CMR characteristics can be seen in Table 2. Change in indexed $\mathrm{LV}$ mass was similar between the two groups (nQRS $-15.9 \pm 10.4$ vs LBBB-T $-13.3 \pm 9.6 \mathrm{~g} / \mathrm{m}^{2}, p=$ 0.367 ) as was change in LVEDVi (nQRS $-7.3 \pm 17.4$ vs LBBB-T $\left.-3.2 \pm 14.5 \mathrm{ml} / \mathrm{m}^{2}, p=0.373\right)$. Neither group experienced any change in right ventricular longitudinal function (nQRS $21.7 \pm 7.0$ to $21.5 \pm 6.2 \mathrm{~mm}, p=$ 0.817 , LBBB-T $18.9 \pm 5.8$ to $18.6 \pm 5.8 \mathrm{~mm}, p=0.773$ ). Post-procedure aortic regurgitant fraction was similar between groups (nQRS $5.4 \pm 5.7$ vs LBBB-T $5.5 \pm 3.3 \%$, $p=0.948$ ). There was a significant correlation between change in QRS and change in LVEF $(r=-0.434, p=$ 0.002 ) and between change in QRS and change in GLS $(r=0.462, p=0.001)$. The relationship between post- 
Table 2 CMR parameters pre and $6 \mathrm{~m}$ post-TAVR according to post-procedure QRS status

\begin{tabular}{|c|c|c|c|}
\hline & $\begin{array}{l}\mathrm{nQRS} \\
(n=24)\end{array}$ & $\begin{array}{l}\text { LBBB-T } \\
(n=24)\end{array}$ & $P$ Value \\
\hline \multicolumn{4}{|c|}{ Left ventricular ejection fraction } \\
\hline Pre-procedure & $54.1 \pm 11.5$ & $56.6 \pm 10.5$ & 0.386 \\
\hline Post-procedure & $58.7 \pm 9.0$ & $54.4 \pm 9.3$ & 0.070 \\
\hline$P$ Value & 0.010 & 0.092 & \\
\hline \multicolumn{4}{|c|}{ Global longitudinal strain, \% } \\
\hline Pre-procedure & $-15.6 \pm 3.9$ & $-16.2 \pm 4.2$ & 0.638 \\
\hline Post-procedure & $-17.7 \pm 2.7$ & $-15.9 \pm 3.4$ & 0.053 \\
\hline$P$ Value & 0.009 & 0.771 & \\
\hline \multicolumn{4}{|c|}{ Indexed left ventricular mass, $\mathrm{g} / \mathrm{m}^{2}$} \\
\hline Pre-intervention & $74.3 \pm 14.7$ & $73.3 \pm 17.4$ & 0.650 \\
\hline Post-intervention & $58.4 \pm 12.6$ & $60.0 \pm 13.7$ & 0.665 \\
\hline$P$ Value & $<0.001$ & $<0.001$ & \\
\hline \multicolumn{4}{|c|}{ Indexed left ventricular end diastolic volume, $\mathrm{ml} / \mathrm{m}^{2}$} \\
\hline Pre-intervention & $97.8 \pm 22.8$ & $93.4 \pm 22.1$ & 0.500 \\
\hline Post-intervention & $90.5 \pm 21.0$ & $90.3 \pm 21.0$ & 0.968 \\
\hline$P$ Value & 0.051 & 0.298 & \\
\hline \multicolumn{4}{|c|}{ Indexed left ventricular end systolic volume, $\mathrm{ml} / \mathrm{m}^{2}$} \\
\hline Pre-intervention & $46.6 \pm 20.4$ & $41.8 \pm 17.7$ & 0.458 \\
\hline Post-intervention & $38.7 \pm 16.2$ & $42.4 \pm 17.8$ & 0.523 \\
\hline$P$ Value & 0.011 & 0.886 & \\
\hline \multicolumn{4}{|c|}{ Indexed left ventricular stroke volume, $\mathrm{ml} / \mathrm{m}^{2}$} \\
\hline Pre-intervention & $51.2 \pm 10.3$ & $51.4 \pm 10.5$ & 0.945 \\
\hline Post-intervention & $51.8 \pm 8.7$ & $47.9 \pm 8.5$ & 0.122 \\
\hline$P$ Value & 0.742 & 0.035 & \\
\hline \multicolumn{4}{|c|}{ Indexed left atrial volume, $\mathrm{ml} / \mathrm{m}^{2}$} \\
\hline Pre-intervention & $67.9 \pm 19.2$ & $72.9 \pm 23.3$ & 0.232 \\
\hline Post-intervention & $60.0 \pm 18.2$ & $67.9 \pm 23.8$ & 0.199 \\
\hline$P$ Value & 0.002 & 0.180 & \\
\hline
\end{tabular}

nQRS narrow QRS post-procedure, LBBB-T new LBBB post-procedure

procedure QRS duration and change in LVESVi, LVEF and GLS can be seen in Fig. 2.

\section{Inter- and intra-ventricular dyssynchrony}

As a group as a whole baseline inter- and intra-ventricular dyssynchrony was $68 \pm 62$ and $54 \pm 83$ ms respectively. Those that subsequently developed LBBB demonstrated more interventricular dyssynchrony at baseline but had similar baseline intraventricular dyssynchrony (Inter: LBBB-T $88 \pm 61$ vs. $\mathrm{nQRS} 48 \pm 57 \mathrm{~ms}, p=0.021$, Intra: LBBB-T $74 \pm 90$ vs. nQRS $35 \pm 73 \mathrm{~ms}, p=0.108)$. In the nQRS group, TAVR was not associated with an improvement in dyssynchrony (Inter: pre-TAVR $47 \pm 57$ vs. postTAVR $23 \pm 86 \mathrm{~ms}, p=0.174$, Intra: pre-TAVR $35 \pm 73$ vs. post-TAVR $7 \pm 102 \mathrm{~ms}, p=0.207$ ). There was evidence of significant inter- and intra-ventricular dyssynchrony in the
LBBB-T group at $6 \mathrm{~m}$ compared with the $\mathrm{nQRS}$ population (Inter: LBBB-T $130 \pm 73$ vs. nQRS $23 \pm 86 \mathrm{~ms}, p<$ 0.001 , intra: LBBB-T $118 \pm 103$ vs. nQRS $13 \pm 13 \mathrm{~ms}, p=$ 0.001 ). There was a significant correlation between postprocedure QRS duration and inter- and intra-ventricular dyssynchrony $(r=0.57, p<0.001$ and $r=0.49, p=<0.001$ respectively). A typical LV contraction pattern in $\mathrm{nQRS}$ and LBBB-T can be seen in Fig. 3.

\section{Predictors of change in LVEF and change in GLS}

Baseline variables which may affect cardiac reverse remodeling following TAVR (including clinical, baseline CMR characteristics and post-procedural aortic regurgitation) were analysed to determine univariable predictors of change in LVEF and GLS (Table 3). Baseline LVEF (beta $-0.414, p=$ 0.015 ) and post-procedure QRS (beta $-0.422, p=<0.001$ ) were independent predictors of change in LVEF at $6 \mathrm{~m}$ on multiple regression analysis. Baseline LVEF (beta $=-0.502$, $p=0.001$ ), baseline GLS (beta $-1.02, p=<0.001$ ) and postprocedure QRS (beta $=0.322, p=0.001$ ) were independent predictors of a change in GLS at $6 \mathrm{~m}$. Infarct pattern LGE at baseline did not impact on post-procedural change in LVEF or change in GLS on univariate analysis.

\section{Discussion}

This is the first study using CMR to investigate the impact of TAVR-induced LBBB on cardiac reverse remodeling in a matched population. The main findings of this study are 1) Those with a narrow QRS post-TAVR have better LVEF and GLS compared to those with LBBB-T $6 \mathrm{~m}$ post-procedure, 2) Patients with LBBB-T exhibited significant inter- and intra-ventricular dyssynchrony compared with those with narrow QRS and 3) Postprocedure QRS duration remained a significant independent predictor of change in LVEF and GLS following TAVR on multivariable analysis.
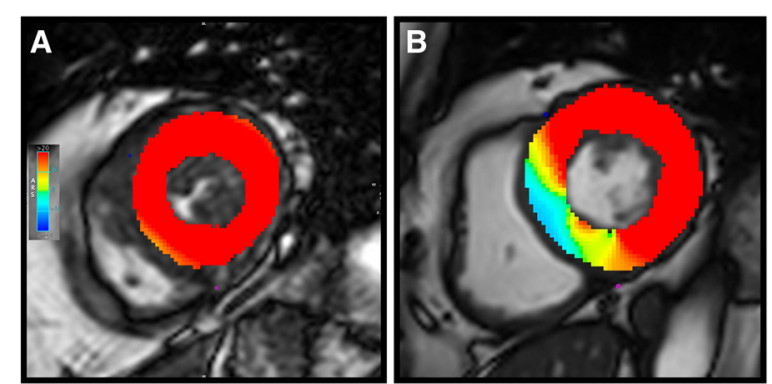

Fig. 3 Radial strain in a single mid-ventricular short axis cine. Panel a shows the typical contraction pattern in a patient with a nQRS, the red colour depicts positive radial strain occurring in all segments of the left ventricle at end systole. Panel $\mathbf{b}$ depicts radial strain at end systole in a patient with TAVR-induced LBBB. Peak positive septal radial strain occurs in early systole and therefore by end-systole the septum is relaxing, depicted by the blue colour 
Table 3 Univariate and multiple regression analysis

\begin{tabular}{|c|c|c|c|c|c|c|}
\hline & Coefficient B & Standard Error & $P$ Value & Coefficient B & Standard error & P Value \\
\hline & \multicolumn{3}{|c|}{ Univariate analysis - change in LVEF } & \multicolumn{3}{|c|}{ Multiple regression analysis - change in LVEF } \\
\hline Age & -0.201 & 0.141 & 0.160 & & & \\
\hline Sex & 2.844 & 2.246 & 0.212 & & & \\
\hline Diabetes mellitus & -1.092 & 2.624 & 0.679 & & & \\
\hline Infarct pattern LGE at baseline & 1.647 & 2.819 & 0.562 & & & \\
\hline STS PROM & -0.020 & 0.448 & 0.965 & & & \\
\hline Post-procedure QRS duration & -0.119 & 0.034 & 0.001 & -0.110 & 0.028 & $<0.001$ \\
\hline AVAi & 7.888 & 14.638 & 0.593 & & & \\
\hline Baseline GLS & -0.963 & 0.249 & $<0.001$ & -0.292 & 0.319 & 0.365 \\
\hline Baseline LVEF & -0.393 & 0.088 & $<0.001$ & -0.295 & 0.117 & 0.015 \\
\hline Baseline fibrosis mass & -0.007 & 0.242 & 0.975 & & & \\
\hline \multirow[t]{2}{*}{ Post procedure aortic regurgitation fraction } & 0.089 & 0.252 & 0.725 & & & \\
\hline & \multicolumn{3}{|c|}{ Univariate analysis - change in GLS } & \multicolumn{3}{|c|}{ Multiple regression analysis - change in GLS } \\
\hline Age & 0.090 & 0.064 & 0.167 & & & \\
\hline Sex & -1.161 & 1.028 & 0.265 & & & \\
\hline Diabetes mellitus & -0.467 & 1.197 & 0.698 & & & \\
\hline Infarct pattern LGE at baseline & -0.078 & 1.291 & 0.952 & & & \\
\hline STS PROM & -0.108 & 0.204 & 0.600 & & & \\
\hline Post-procedure QRS duration & 0.044 & 0.016 & 0.010 & 0.038 & 0.011 & 0.001 \\
\hline AVAi & -4.954 & 6.658 & 0.461 & & & \\
\hline Baseline GLS & -0.588 & 0.098 & $<0.001$ & -0.904 & 0.122 & $<0.001$ \\
\hline Baseline LVEF & 0.094 & 0.046 & 0.046 & -0.163 & 0.044 & 0.001 \\
\hline Post-procedure aortic regurgitation fraction & -0.015 & 0.116 & 0.895 & & & \\
\hline Baseline fibrosis mass & -0.004 & 0.112 & 0.970 & & & \\
\hline
\end{tabular}

LVEF left ventricular ejection fraction, LGE late gadolinium enhancement, STS PROM society of thoracic surgeons predicted risk of mortality, AVAi indexed aortic valve area, GLS global longitudinal strain

\section{Impact of TAVR-induced LBBB on cardiac reverse remodeling}

TAVR-induced LBBB is common, occurring in 16-65\% patients depending on valve type [2]. Although predictors of LBBB-T have been extensively studied [2], the impact of LBBB-T on cardiac reverse remodeling is less well described, with studies limited to echocardiographic evaluation and containing a heterogeneous mix of patients. A PARTNER echocardiographic sub-study reported a lower LVEF at 12 months in patients with LBBB on discharge electrocardiogram compared to those with a narrow QRS, however, there was an increased number of those undergoing trans-apical TAVR in the LBBB-T group [6], findings which were replicated in another similar study, again with more undergoing transapical TAVR in the LBBB-T group [20]. Tzikas et al. [10] reported an $8 \%$ difference in LVEF between those with LBBB-T and nQRS prior to and 6 days following selfexpanding TAVR. Longitudinal strain was also nonsignificantly reduced in those with new conduction abnormalities. Hoffman et al. [11] investigated 90 patients using 2D and speckle tracking trans-thoracic echocardiography prior to and at 1 and 12 months following TAVR. Patients with new conduction defects had a significantly larger LVESVi at 12 months compared with those with a narrow QRS, with less difference in LVEDVi, mirroring the findings in our study. New conduction defects and baseline LVEF were independent predictors of reduction in LVEF at 12 months. The inclusion of patients with trans-apical access in the majority of these studies $[6,11,20]$ and those with post-procedural pacemaker insertion $[6,10,11,20]$ is a significant confounder, however, given that transapical access has been linked to reduced LVEF in a number of studies $[20,21]$ and pacing induced LBBB has been shown to cause different patterns of strain to those with idiopathic LBBB [22].

Our study adds further insight into the impact of LBBB-T on cardiac reverse remodeling. The accuracy and reproducibility of $\mathrm{CMR}$ means that important 
differences can be determined using studies $87 \%$ smaller than echocardiographically based studies, with only 11 patients per group required to detect a 3\% difference in LVEF [13]. Our study groups were matched for clinical and baseline CMR characteristics, all parameters which have been found to strongly influence reverse remodeling following valve intervention [16]. None of the patients in our study received trans-apical TAVR or permanent pacemaker insertion and the unique ability of CMR LGE imaging allowed us to identify and exclude any patients who had a post-procedural myocardial infarction, another factor that may have confounded the earlier echocardiographically based studies. Finally, the two groups experienced similar amounts of postprocedural aortic regurgitation, which is an important modulator of post-TAVR reverse remodeling [23, 24], and which was not reported in most of the echocardiographic studies $[6,10,11]$.

\section{Inter and intra-ventricular dyssynchrony}

The novel use of CMR feature tracking allows us to report values for intra- and inter-ventricular dyssynchrony. In LBBB, the normally functioning right bundle conducts the electrical impulse to the right ventricle prompting early right ventricular contraction followed by activation of the interventricular septum and finally lateral wall contraction resulting in inter- and intraventricular dyssynchrony. This dyssynchrony leads to the classical abnormal septal motion pattern of contraction seen in LBBB which is felt to impair LV filling and ejection in its own right (Fig. 3). This dysynchronous contraction leads to an increase in LVESVi, as seen in our LBBB-T group and it is this, rather than a change in LVEDVi that is the largest driver of reduction in LVEF. We have also shown that LBBB-T impacted on change in GLS, with no improvement in this group compared to a significant improvement in the nQRS group. Although GLS may be affected by dyssynchrony [25], this, coupled with the reduction in left atrial volume in the $\mathrm{nQRS}$ but not the LBBB-T group, and the reduction in LV stroke volume in those with $L B B B-T$, suggests that the effects of LBBB-T may go beyond that of simple mechanical dyssynchrony.

\section{Conduction system damage during TAVR}

It is well established that TAVR leads to conduction abnormalities [2]. Trauma can occur at multiple timepoints during the TAVR procedure; from guidewire manipulation, to during balloon valvuloplasty, device manipulation and valve deployment. It is likely that the different valve designs can cause differing degrees of compression to the conduction system; with the self-expanding Medtronic CoreValve felt to cause more compression of the LV outflow tract than the balloon expandable Edwards Sapien device [26]. The unique design of the mechanically expandable and repositionable Boston Lotus valve with its adaptive seal, may also be associated with more conduction system trauma, although reports to date are limited [27]. Global ischaemia during rapid pacing required for valve deployment may exacerbate the issue [2]. Other procedure-related factors felt to be implicated include pre-implant valvuloplasty, deep implant, low ratio of the annulus:balloon or annulus:prosthesis and operator experience [28].

\section{Clinical implications}

The impact of TAVR-induced LBBB on mortality is a subject of debate, however, it has been shown in many studies to be a predictor of mortality $[3-5,29]$ and has been associated with increased hospitalisation [6]. Other studies have failed to demonstrate a link [12, 20, 30]. Nonetheless, LVEF is a strong independent predictor of long term survival [31]. Our study has shown that TAVR-induced LBBB is associated with reduced global longitudinal and radial systolic function compared with those with a narrow post-procedure QRS, which could partially explain the link with mortality. Given the adverse effect of TAVR-induced LBBB on cardiac reverse remodeling, restoring inter- and intra-ventricular dyssynchrony using cardiac resynchronisation therapy, could be considered, especially if another conventional indication for device therapy exists. Furthermore, every effort should be made by the operator to reduce the risk of TAVR-induced LBBB given the adverse effects on ventricular remodeling seen. As newer devices are being developed, designs should be focused on minimising damage to the electrical conducting system in order to prevent the deleterious effects on the LV that this entails.

\section{Study limitations}

Although patients were recruited in a prospective manner, they were matched retrospectively and hence the study is prone to the selection bias of this type of study. Patients with LBBB-T were matched according to those factors known to influence cardiac reverse remodeling [16] but other factors may be unaccounted for. Specifically, patients with coronary artery disease and previous myocardial infarction were included in the study, however, numbers in each group were similar and infarct pattern LGE at baseline was not a univariate predictor of change in LVEF or GLS. Group allocation was based on the discharge electrocardiogram and not re-confirmed at 6 months, however, there are evidence to suggest that virtually all those with $\angle B B B$ at discharge have persistent LBBB at 30 days following self-expanding TAVR [30]. Furthermore, the demonstration of ongoing dyssynchrony at $6 \mathrm{~m}$ in the LBBB-T group suggests that the 
conduction abnormality was persistent. Finally, although adequately powered to detect a difference in reverse remodeling using the accurate technique of CMR, the study is small with a relatively short follow-up period and a larger study with a longer follow-up interval may be helpful to further investigate the impact of TAVRinduced LBBB on cardiac reverse remodeling.

\section{Conclusion}

New LBBB following TAVR is associated with less favourable cardiac reverse remodeling, including effects on LVEF, global longitudinal strain and inter- and intraventricular dyssynchrony. In view of this, every effort should be made to minimise the risk of TAVR-induced LBBB especially as TAVR is now being extended to a younger, lower risk population.

\section{Abbreviations}

CMR: Cardiovascular magnetic resonance; GLS: Global longitudinal strain; LBBB: Left-bundle branch block; LBBB-T: TAVR-induced left bundle branch block; LGE: Late gadolinium enhancement; LV: Left ventricular; LVEDVi: Indexed left ventricular end diastolic volume; LVEF: Left ventricular ejection fraction; LVESVi: Indexed left ventricular end systolic volume; nQRS: Narrow QRS; TAVR: Trans-catheter aortic valve replacement

\section{Acknowledgements}

The authors are grateful for the support and assistance of the research nurses (Fiona Richards, Petra Bijsterveld and Lisa Clark) and the radiographers (Gavin Bainbridge, Caroline Richmond and Margaret Saysell) during this project.

\section{Funding}

TAM is funded by a British Heart Foundation (BHF) Project Grant (PG/11/126/ 29321); PPS is funded by BHF Clinical Fellowship (FS/12/88/29474); SP is funded by BHF Senior Research Fellowship (FS/10/62/28409). This study was part-funded by the NIHR Leeds Clinical Research Facility. The views expressed are those of the author(s) and not necessarily those of the NHS, NIHR or the Department of Health.

\section{Availability of data and materials}

This can be provided on request.

\section{Authors' contribution}

L.D: conception, design, patient recruitment, collection of data, data analysis and interpretation of data, drafting and revision of manuscript; T.M: patient recruitment, collection of data and revision of manuscript, A.U: patient recruitment, collection of data and revision of manuscript, T.F: patient recruitment, collection of data and revision of manuscript, O.B: Collection of data, data analysis and revision of manuscript, P.S: analysis and interpretation of data and revision of manuscript, P.H: collection of data, data analysis and revision of manuscript, J.F: collection of data and revision of manuscript, P.G: collection of data and revision of manuscript, G.F: collection of data and revision of manuscript, C.M: study design, collection of data and revision of manuscript, D.B: conception, design, collection of data and revision of manuscript, S.P: study design and revision of manuscript, J.G: conception, design, collection and interpretation of data, drafting and revision of manuscript. All authors read and approved the final manuscript.

\section{Competing interests}

JPG and SP have received an educational research grant from Philips Healthcare. DB and CJM are consultants and proctors for both Medtronic and Boston Scientific.

Consent for publication

Consent for publication has been obtained from all patients enrolled in the study.

\section{Ethics approval and consent to participate}

This study was approved by the Leeds (west) Research Ethics Committee (08/H1307/106) and complied with the declaration of Helsinki. All patients provided written informed consent.

Received: 24 January 2017 Accepted: 2 February 2017

Published online: 22 February 2017

References

1. Poels $\pi$, Houthuizen P, Van Garsse LA, et al. Frequency and prognosis of new bundle branch block induced by surgical aortic valve replacement. Eur J Cardiothorac Surg. 2015;47:e47-53.

2. van der Boon RM, Nuis RJ, Van Mieghem NM, et al. New conduction abnormalities after TAVI-frequency and causes. Nat Rev Cardiol. 2012;9:454-63.

3. Houthuizen P, Van Garsse LA, Poels TT, et al. Left bundle-branch block induced by transcatheter aortic valve implantation increases risk of death. Circulation. 2012;126:720-8.

4. Schymik G, Tzamalis $P$, Bramlage $P$, et al. Clinical impact of a new left bundle branch block following TAVI implantation: 1-year results of the TAVIK cohort. Clin Res Cardiol. 2015:104:351-62.

5. Meguro K, Lellouche N, Yamamoto M, et al. Prognostic value of QRS duration after transcatheter aortic valve implantation for aortic stenosis using the CoreValve. Am J Cardiol. 2013;111:1778-83.

6. Nazif TM, Williams MR, Hahn RT, et al. Clinical implications of new-onset left bundle branch block after transcatheter aortic valve replacement: analysis of the PARTNER experience. Eur Heart J. 2014:35:1599-607.

7. Zannad F, Huvelle E, Dickstein $K$, et al. Left bundle branch block as a risk factor for progression to heart failure. Eur J Heart Fail. 2007;9:7-14.

8. Cinca J, Mendez A, Puig T, et al. Differential clinical characteristics and prognosis of intraventricular conduction defects in patients with chronic heart failure. Eur J Heart Fail. 2013;15:877-84.

9. Urena M, Webb JG, Eltchaninoff $\mathrm{H}$, et al. Late cardiac death in patients undergoing transcatheter aortic valve replacement: incidence and predictors of advanced heart failure and sudden cardiac death. J Am Coll Cardiol. 2015:65:437-48.

10. Tzikas A, van Dalen BM, Van Mieghem NM, et al. Frequency of conduction abnormalities after transcatheter aortic valve implantation with the Medtronic-CoreValve and the effect on left ventricular ejection fraction. Am J Cardiol. 2011;107:285-9.

11. Hoffmann R, Herpertz R, Lotfipour S, et al. Impact of a new conduction defect after transcatheter aortic valve implantation on left ventricular function. JACC Cardiovasc Interv. 2012;5:1257-63.

12. Carrabba N, Valenti R, Migliorini A, et al. Impact on Left Ventricular Function and Remodeling and on 1-Year Outcome in Patients With Left Bundle Branch Block After Transcatheter Aortic Valve Implantation. Am J Cardiol. 2015:116:125-31.

13. Grothues F, Smith GC, Moon JC, et al. Comparison of interstudy reproducibility of cardiovascular magnetic resonance with two-dimensional echocardiography in normal subjects and in patients with heart failure or left ventricular hypertrophy. Am J Cardiol. 2002:90:29-34.

14. Onishi T, Saha SK, Delgado-Montero A, et al. Global longitudinal strain and global circumferential strain by speckle-tracking echocardiography and feature-tracking cardiac magnetic resonance imaging: comparison with left ventricular ejection fraction. J Am Soc Echocardiogr. 2015;28:587-96.

15. Vahanian A, Alfieri O, Andreotti $F$, et al. Guidelines on the management of valvular heart disease (version 2012). Eur Heart J. 2012;33:2451-96.

16. Dobson LE, Fairbairn TA, Musa TA, et al. Sex-related differences in left ventricular reverse remodeling in severe aortic stenosis and reverse remodeling after aortic valve replacement: A cardiovascular magnetic resonance study. Am Heart J. 2016;175:101-11.

17. Willems JL, de Medina EO R, Bernard R, et al. Criteria for intraventricular conduction disturbances and pre-excitation. World Health Organizational/ International Society and Federation for Cardiology Task Force. J Am Coll Cardiol. 1985;5:1261-75.

18. Fine NM, Tandon S, Kim HW, et al. Validation of sub-segmental visual scoring for the quantification of ischemic and nonischemic myocardial fibrosis using late gadolinium enhancement MRI. J Magn Reson Imaging. 2013:38:1369-76.

19. Gulati A, Ismail TF, Jabbour A, et al. Clinical utility and prognostic value of left atrial volume assessment by cardiovascular magnetic resonance in nonischaemic dilated cardiomyopathy. Eur J Heart Fail. 2013;15:660-70. 
20. Urena M, Webb JG, Cheema A, et al. Impact of new-onset persistent left bundle branch block on late clinical outcomes in patients undergoing transcatheter aortic valve implantation with a balloon-expandable valve. JACC Cardiovasc Interv. 2014;7:128-36.

21. Meyer CG, Frick M, Lotfi S, et al. Regional left ventricular function after transapical vs. transfemoral transcatheter aortic valve implantation analysed by cardiac magnetic resonance feature tracking. Eur Heart J Cardiovasc Imaging. 2014;15:1168-76.

22. Park HE, Kim JH, Kim HK, et al. Ventricular dyssynchrony of idiopathic versus pacing-induced left bundle branch block and its prognostic effect in patients with preserved left ventricular systolic function. Am J Cardiol. 2012; 109:556-62.

23. Poulin F, Carasso S, Horlick EM, et al. Recovery of left ventricular mechanics after transcatheter aortic valve implantation: effects of baseline ventricular function and postprocedural aortic regurgitation. J Am Soc Echocardiogr. 2014;27:1133-42

24. Merten C, Beurich HW, Zachow D, et al. Aortic regurgitation and left ventricular remodeling after transcatheter aortic valve implantation: a serial cardiac magnetic resonance imaging study. Circ Cardiovasc Interv. 2013;6: 476-83.

25. Helm RH, Leclercq C, Faris OP, et al. Cardiac dyssynchrony analysis using circumferential versus longitudinal strain: implications for assessing cardiac resynchronization. Circulation. 2005;111:2760-7.

26. Urena M, Mok M, Serra V, et al. Predictive factors and long-term clinical consequences of persistent left bundle branch block following transcatheter aortic valve implantation with a balloon-expandable valve. J Am Coll Cardiol. 2012;60:1743-52

27. Gooley RP, Talman AH, Cameron JD, Lockwood SM, Meredith IT. Comparison of Self-Expanding and Mechanically Expanded Transcatheter Aortic Valve Prostheses. JACC Cardiovasc Interv. 2015;8:962-71.

28. Poels $T$, Houthuizen P, Van Garsse LA, Maessen JG, de Jaegere P, Prinzen FW. Transcatheter aortic valve implantation-induced left bundle branch block: causes and consequences. J Cardiovasc Transl Res. 2014;7:395-405.

29. Houthuizen $\mathrm{P}$, van der Boon RM, Urena M, et al. Occurrence, fate and consequences of ventricular conduction abnormalities after transcatheter aortic valve implantation. Eurolntervention. 2014;9:1142-50.

30. Testa L, Latib A, De Marco F, et al. Clinical impact of persistent left bundlebranch block after transcatheter aortic valve implantation with CoreValve Revalving System. Circulation. 2013;127:1300-7.

31. Dahl JS, Eleid MF, Michelena HI, et al. Effect of left ventricular ejection fraction on postoperative outcome in patients with severe aortic stenosis undergoing aortic valve replacement. Circ Cardiovasc Imaging. 2015;8: e002917.

\section{Submit your next manuscript to BioMed Central and we will help you at every step:}

- We accept pre-submission inquiries

- Our selector tool helps you to find the most relevant journal

- We provide round the clock customer support

- Convenient online submission

- Thorough peer review

- Inclusion in PubMed and all major indexing services

- Maximum visibility for your research

Submit your manuscript at www.biomedcentral.com/submit

) Biomed Central 